\title{
Evolution of Solar Wind Turbulence from 0.1 to 1 au during the First Parker Solar Probe-Solar Orbiter Radial Alignment
}

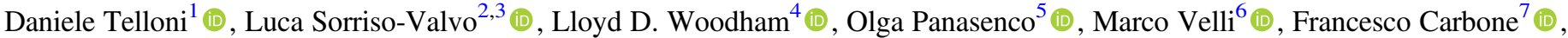

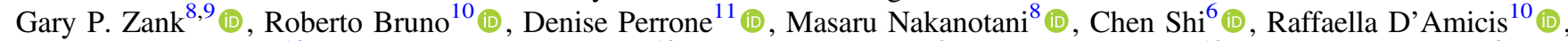

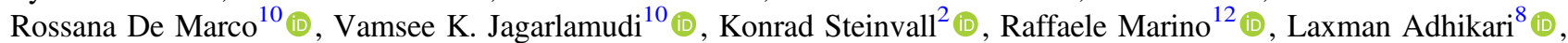

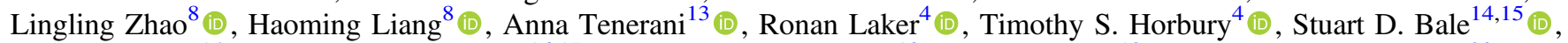 \\ Marc Pulupa $^{14}$ (1), David M. Malaspina ${ }^{16,17}$ (1), Robert J. MacDowall ${ }^{18}$ (1) , Keith Goetz $^{19}$ (1) , Thierry Dudok de Wit ${ }^{20}$ (1),

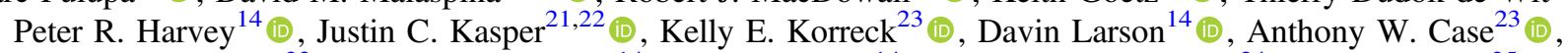 \\ Michael L. Stevens ${ }^{23}$ (1), Phyllis Whittlesey ${ }^{14}$ (D), Roberto Livi ${ }^{14}$ (D), Christopher J. Owen ${ }^{24}$ (1), Stefano Livi ${ }^{25}$ (D), \\ Philippe Louarn $^{26}$ (10), Ester Antonucci ${ }^{1}$ (10), Marco Romoli ${ }^{27}$ (1) , Helen O’Brien ${ }^{4}$ (D), Vincent Evans ${ }^{4}$ (10), and Virginia Angelini ${ }^{4}$ (1) \\ ${ }^{1}$ National Institute for Astrophysics, Astrophysical Observatory of Torino, Via Osservatorio 20, I-10025 Pino Torinese, Italy; daniele.telloni@ inaf.it \\ ${ }^{2}$ Swedish Institute of Space Physics, Ångström Laboratory, Lägerhyddsvägen 1, SE-751 21 Uppsala, Sweden \\ ${ }^{3}$ National Research Council, Institute for the Science and Technology of Plasmas, Via Amendola 122/D, I-70126 Bari, Italy \\ ${ }^{4}$ Department of Physics, Imperial College London, London SW7 2AZ, UK \\ ${ }^{5}$ Advanced Heliophysics, Pasadena, CA 91106, USA \\ ${ }^{6}$ Earth, Planetary, and Space Sciences, University of California, Los Angeles, CA 90095, USA \\ ${ }_{8}^{7}$ National Research Council, Institute of Atmospheric Pollution Research, c/o University of Calabria, I-87036 Rende, Italy \\ ${ }^{8}$ Center for Space Plasma and Aeronomic Research, University of Alabama in Huntsville, Huntsville, AL 35805, USA \\ ${ }^{9}$ Department of Space Science, University of Alabama in Huntsville, Huntsville, AL 35805, USA \\ ${ }^{10}$ National Institute for Astrophysics, Institute for Space Astrophysics and Planetology, Via del Fosso del Cavaliere 100, I-00133 Roma, Italy \\ ${ }^{11}$ Italian Space Agency, Via del Politecnico snc, I-00133 Roma, Italy \\ ${ }^{12}$ Laboratoire de Mécanique des Fluides et d'Acoustique, Centre National de la Recherche Scientifique, École Centrale de Lyon, Université Claude Bernard Lyon 1, \\ INSA de Lyon, F-69134 Écully, France \\ ${ }^{13}$ Department of Physics, The University of Texas at Austin, Austin, TX 78712, USA \\ ${ }^{14}$ Space Sciences Laboratory, University of California, Berkeley, CA 94720, USA \\ ${ }^{15}$ Physics Department, University of California, Berkeley, CA 94720, USA \\ ${ }_{17}^{16}$ Astrophysical and Planetary Sciences Department, University of Colorado, Boulder, CO 80309, USA \\ ${ }^{17}$ Laboratory for Atmospheric and Space Physics, University of Colorado, Boulder, CO 80303, USA \\ ${ }^{18}$ National Aeronautics and Space Administration, Goddard Space Flight Center, Greenbelt, MD 20771, USA \\ ${ }^{19}$ School of Physics and Astronomy, University of Minnesota, Minneapolis, MN 55455, USA \\ ${ }^{20}$ Laboratoire de Physique et de Chimie de l'Environnement et de l'Espace, Center National de la Recherche Scientifique, 3A Avenue de la Recherche Scientifique, \\ F-45071 Orléans, France \\ ${ }^{21}$ BWX Technologies, Inc., Washington, DC 20002, USA \\ ${ }^{22}$ Climate and Space Sciences and Engineering, University of Michigan, Ann Arbor, MI 48109, USA \\ ${ }^{23}$ Harvard-Smithsonian Center for Astrophysics, Cambridge, MA 02138, USA \\ ${ }^{24}$ Mullard Space Science Laboratory, University College London, Holmbury St. Mary, RH5 6NT Dorking, UK \\ ${ }^{25}$ Southwest Research Institute, San Antonio, TX 78238, USA \\ ${ }^{26}$ Institut de Recherche en Astrophysique et Planétologie, Center National de la Recherche Scientifique, Université de Toulouse, 9 Avenue du Colonel Roche, \\ F-31028 Toulouse, France \\ ${ }^{27}$ University of Florence, Department of Physics and Astronomy, Via Giovanni Sansone 1, I-50019 Sesto Fiorentino, Italy \\ Received 2021 March 30; revised 2021 April 14; accepted 2021 April 14; published 2021 May 6
}

\begin{abstract}
The first radial alignment between Parker Solar Probe and Solar Orbiter spacecraft is used to investigate the evolution of solar wind turbulence in the inner heliosphere. Assuming ballistic propagation, two $1.5 \mathrm{hr}$ intervals are tentatively identified as providing measurements of the same plasma parcels traveling from 0.1 to $1 \mathrm{au}$. Using magnetic field measurements from both spacecraft, the properties of turbulence in the two intervals are assessed. Magnetic spectral density, flatness, and high-order moment scaling laws are calculated. The Hilbert-Huang transform is additionally used to mitigate short sample and poor stationarity effects. Results show that the plasma evolves from a highly Alfvénic, less-developed turbulence state near the Sun, to fully developed and intermittent turbulence at 1 au. These observations provide strong evidence for the radial evolution of solar wind turbulence.
\end{abstract}

Unified Astronomy Thesaurus concepts: Magnetohydrodynamics (1964); Alfven waves (23); Space plasmas (1544); Interplanetary turbulence (830); Solar wind (1534)

\section{Introduction}

The interplanetary medium is a continuous, highly variable, weakly collisional plasma outflow from the Sun. In situ spacecraft observations generally reveal the solar wind far away from the Sun to be in a state of fully developed turbulence (Coleman 1968; Bruno \& Carbone 2013). The energy in electromagnetic and velocity fluctuations, injected at the Sun and during the solar wind expansion into the heliosphere, is channeled toward smaller spatial/timescales through a turbulent cascade until it eventually dissipates. The power spectral density of magnetic and velocity fields within the inertial range shows a power-law dependence similar to that observed in fluid turbulence (Kolmogorov 1941; Tu \& Marsch 1995). In the fast solar wind, a low-frequency spectral break, which separates the inertial range from (larger) injection scales, moves to larger and larger scales with distance from the Sun (Bruno \& Carbone 2013). At scales below the inertial range, 
ions become unmagnetized and plasma dynamics is governed by particle kinetic properties. There, another spectral break is observed, whose frequency also depends on radial distance from the Sun, consistently with proton cyclotron resonance conditions (Bruno \& Trenchi 2014). In addition to spectral breaks, other properties of turbulence (such as Alfvénic content, intermittency, and anisotropy) also evolve with heliocentric distance (see the review by Bruno \& Carbone 2013, and references therein).

One way to study the radial evolution of solar wind turbulence properties is to consider plasma coming from the same solar source. A well-defined high-speed stream observed by Helios at different heliodistances during three consecutive solar rotations was used to study the radial evolution of turbulence (Bavassano et al. 1981, 1982a, 1982b; He et al. 2013) and intermittency (Bruno et al. 2003, 2014a) in the inner heliosphere. More recently, by means of reprocessed particle data from the Helios mission, 16 intervals of unperturbed highspeed plasma, from three different coronal holes and at different radial distances between 0.3 and $1 \mathrm{au}$, have been identified to investigate the large-scale physics and thermodynamics of the solar wind (Perrone et al. 2019a, 2019b).

An ideal (although rarely used) approach is to follow the same plasma parcel during its expansion. This method requires two or more radially aligned spacecraft at different distances. The first instance of these exceptional cases occurred when the Helios 1 and Helios 2 sampled the same high-speed stream plasma at $\sim 0.51$ and $\sim 0.72 \mathrm{au}$, respectively (Schwartz \& Marsch 1983). The authors found clear evidence that particle adiabatic invariants are not conserved, suggesting some ion perpendicular heating. Successive alignments in the inner heliosphere, between Wind and MESSENGER (Bruno \& Trenchi 2014; Bruno et al. 2014b; Telloni et al. 2015), and in the outer heliosphere, between Wind and Ulysses (Bruno \& Trenchi 2014) or ACE and Ulysses (D'Amicis et al. 2010), were used to study the evolution of the solar wind turbulence between $\sim 0.4$ and $\sim 5.3$ au systematically. These studies showed that the ion spectral break moves to higher and higher frequencies approaching the Sun (D'Amicis et al. 2010; Bruno $\&$ Trenchi 2014). In addition, Bruno et al. (2014b) found that the spectral slope of the dissipative range depends strongly on the power associated with the fluctuations within the inertial range, i.e., the higher the power, the steeper the slope. Finally, the high-frequency magnetic fluctuations beyond the spectral break show a character compatible with left-handed ion cyclotron waves and right-handed kinetic Alfvén waves, with the low-frequency limit of these fluctuations following the radial evolution of the ion spectral break (Telloni et al. 2015).

Thanks to the new inner heliospheric missions of the Parker Solar Probe (PSP; Fox et al. 2016), launched in 2018 August, and the Solar Orbiter (SolO, Müller et al. 2020), launched in 2020 February, a new golden era for solar wind physics is upon us. PSP is observing a completely unexplored environment close to the Sun, while SolO will combine both highresolution in situ measurements and remote-sensing observations (Zouganelis et al. 2020) aiming to accurately determine magnetic connectivity between the solar wind plasma and its source regions at the Sun. Indeed, the synergy between the two spacecraft allows for a detailed mapping of the inner heliosphere (Velli et al. 2020). In particular, radial alignments between the two probes allow the study of the evolution of the solar wind at distances never reached before. The first line-up between PSP and SolO occurred in 2020 September, when PSP (approaching perihelion during its sixth solar encounter) and SolO were orbiting at $\sim 0.1$ and $\sim 1$ au, respectively.

The aim of the present Letter is to exploit this PSP-Solo radial alignment to study, for the first time, the radial evolution of the solar wind turbulence properties, namely magnetic spectra, compressibility and intermittency, from near the Alfvén radius (Goelzer et al. 2014) out to Earth orbit.

\section{PSP-SolO Radial Alignment Identification}

The identification of the time intervals corresponding to the same plasma parcel observed at PSP and SolO distances during their radial alignment with the Sun is based on the ballistic approach sketched in the cartoon of Figure 1 (top panel). The condition for radial alignment requires that the longitude at PSP, $\Phi_{\mathrm{PSP}}$, at the instant $t_{0}$ when the spacecraft samples the plasma parcel is equal to that at SolO, $\Phi_{\text {SolO }}$, at the instant $t_{0}+\Delta t$, where $\Delta t$ is the plasma transit time from PSP to SolO, i.e.,

$$
\begin{aligned}
\Phi_{\mathrm{PSP}}\left(t_{0}\right) & =\Phi_{\mathrm{SolO}}\left[t_{0}+\Delta t\right] \\
& =\Phi_{\mathrm{SolO}}\left[t_{0}+\Delta R / V_{\mathrm{PSP}}\left(t_{0}\right)\right] \\
& =\Phi_{\mathrm{SolO}}\left[t_{0}+\left(R_{\mathrm{SolO}}\left(t_{0}+\Delta t\right)-R_{\mathrm{PSP}}\left(t_{0}\right)\right) / V_{\mathrm{PSP}}\left(t_{0}\right)\right],
\end{aligned}
$$

where $\Delta R$ is the radial separation between PSP and SolO, located at $R_{\mathrm{PSP}}$ and $R_{\mathrm{SolO}}$, respectively, and $V_{\mathrm{PSP}}$ is the solar wind speed observed at PSP.

On the basis of the PSP and SolO ephemerides and the plasma speed observed at PSP (in the inertial Radial Tangential Normal (RTN) frame) by the SPAN-Ai top-hat electrostatic analyzer (part of the SWEAP suite on board PSP, Kasper et al. 2016), it follows that the plasma crossing PSP on 2020 September 27 (at $\simeq 04: 00$ UT) could be sampled by SolO on 2020 October 2 (around $\simeq 00: 00$ UT). Applying a tolerance of \pm 1.5 longitude for the radial alignment, time intervals of $1.5 \mathrm{hr}$ centered on the above times are thus identified as corresponding to the same plasma parcels.

From Equation (1) one sees that the timings relative to the radial alignment depend on $V_{\mathrm{PSP}}$ and possible subsequent acceleration, especially for SolO. The extension of the magnetic parcel under study is so short (hours) with respect to the much longer transit time (days) that uncertainties related to plasma velocity measurements (e.g., moments of ion distribution functions include the contribution from the proton beam, which can be very large at times) as well as velocity field fluctuations (from turbulence and/or switchbacks) may easily lead to misalignments. In order to address this issue and check the goodness of the selected time intervals, a complementary strategy has been adopted. The plasma speed at PSP is assumed to range from 250 to $350 \mathrm{~km} \mathrm{~s}^{-1}$ in steps of $1 \mathrm{~km} \mathrm{~s}^{-1}$. From $N=100$ different propagation speeds, $N$ different pairs of $1.5 \mathrm{hr}$ time intervals at PSP and SolO, relative to each potential radial alignment, are thus identified. The pair of time intervals for which the cross-correlation between the magnetic field magnitude $|\boldsymbol{B}|$ measured at PSP (by the fluxgate magnetometer of the FIELDS suite; Bale et al. 2016) and SolO (by the MAG magnetometer; Horbury et al. 2020) maximizes is assumed to be the one corresponding to the same solar wind plasma observed at 0.1 and $1 \mathrm{au}$. The magnetic field magnitude in the same plasma parcel may indeed be expected to be relatively well correlated when observed at different distances, since in situ driving of turbulence may balance dissipation (the reader 

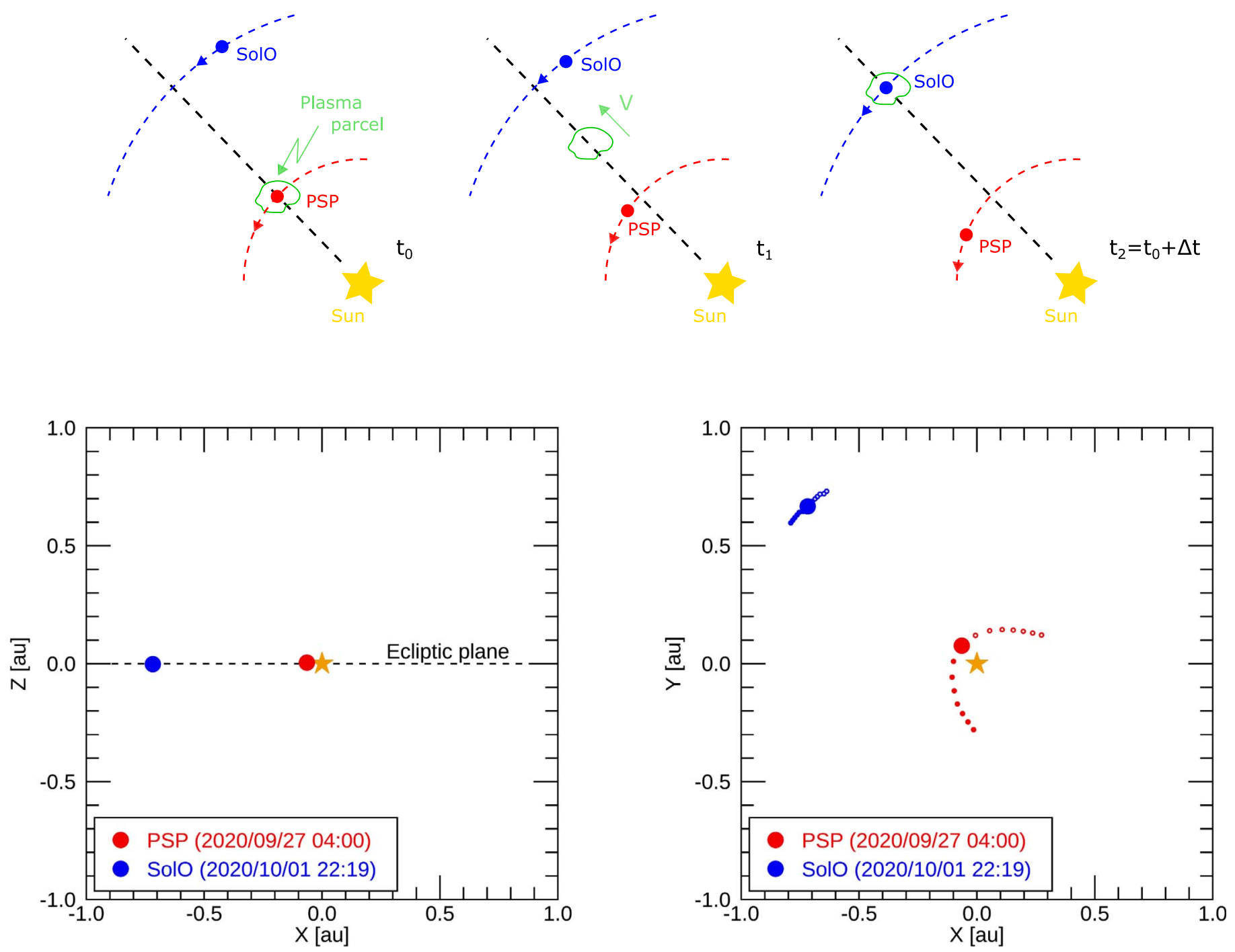

Figure 1. Top panel: cartoon of the encounters of the same plasma parcel with PSP and SolO during their radial alignment. Bottom panels: positions of PSP (red) and SolO (blue) relative to the Sun (yellow star), in the $X Z$ (left) and $X Y$ (right) planes of the heliocentric ecliptic coordinate frame, at the times of the crossing of the same solar wind plasma. The positions the probes had in the seven days before and after the radial alignment are also shown, as empty and full points of smaller size, respectively.

is, e.g., referred to Zank et al. 2017, for the relevant theory). The time intervals with the highest correlation in $|\boldsymbol{B}|$ (0.72 at 0-lag) are found to be 2020 September 27 03:15-04:45 UT at PSP and 2020 October 1 21:34-23:04 UT at SolO, corresponding to a solar wind bulk speed of $320 \mathrm{~km} \mathrm{~s}^{-1}$ at PSP. Both the temporal localization of the parcel and the wind speed at PSP are in good agreement with the independent estimate based on the measured speed at PSP $\left(315 \mathrm{~km} \mathrm{~s}^{-1}\right)$, showing the robustness of the correlation analysis.

The bottom panels of Figure 1 show side (left panel) and top (right panel) views of the ecliptic plane during the PSP-Solo line-up, when the two spacecraft had a radial separation of $0.9 \mathrm{au}$, with PSP approaching perihelion close to $0.1 \mathrm{au}$, i.e., $20 R_{\odot}$, and SolO orbiting at a radial distance of about 1 au.

Unfortunately, during the radial alignment, plasma measurements from SolO were unavailable, thus preventing checks of the solar wind speed at SolO. In order to address this shortcoming, the DSCOVR speed data were time-shifted by about +9.3 days (which accounts for the solar rotation and the different longitude of SolO and DSCOVR). Despite the approximate approach, the resulting expected speed at SolO during the radial alignment is about $324 \mathrm{~km} \mathrm{~s}^{-1}$, only a slight increase with respect to that observed at PSP, confirming the quality of the PSP-SolO radial alignment identification.

Finally, in order to check whether the plasma observed by PSP and $\mathrm{SolO}$ in the selected intervals comes from the same source region at the Sun (further corroborating the above findings) the coronal origins of the solar wind observed by PSP on 2020 September 27 and by SolO on 2020 October 2 have been assessed. Spacecraft positions have been projected onto the source surface and then down to the height of $1.16 R_{\odot}$, using a Potential Field Source Surface (PFSS) model (Schrijver \& De Rosa 2003), as displayed in Figure 2. The source surface height has been adjusted to $2.0 R_{\odot}$, following the magnetic field polarity changes observed by SolO on 2020 September 27 (left panel of Figure 2). A detailed description of the solar wind source mapping can be found in Panasenco et al. (2020). As follows from the comparison between the panels of Figure 2, during the time periods corresponding to the radial alignment, PSP (left panel, in blue) and SolO (right panel, in orange) were connected to the same equatorial extension of the southern polar coronal hole at $240^{\circ}-$ $250^{\circ}$ Carrington Longitude. On 2020 September 27 PSP, at 

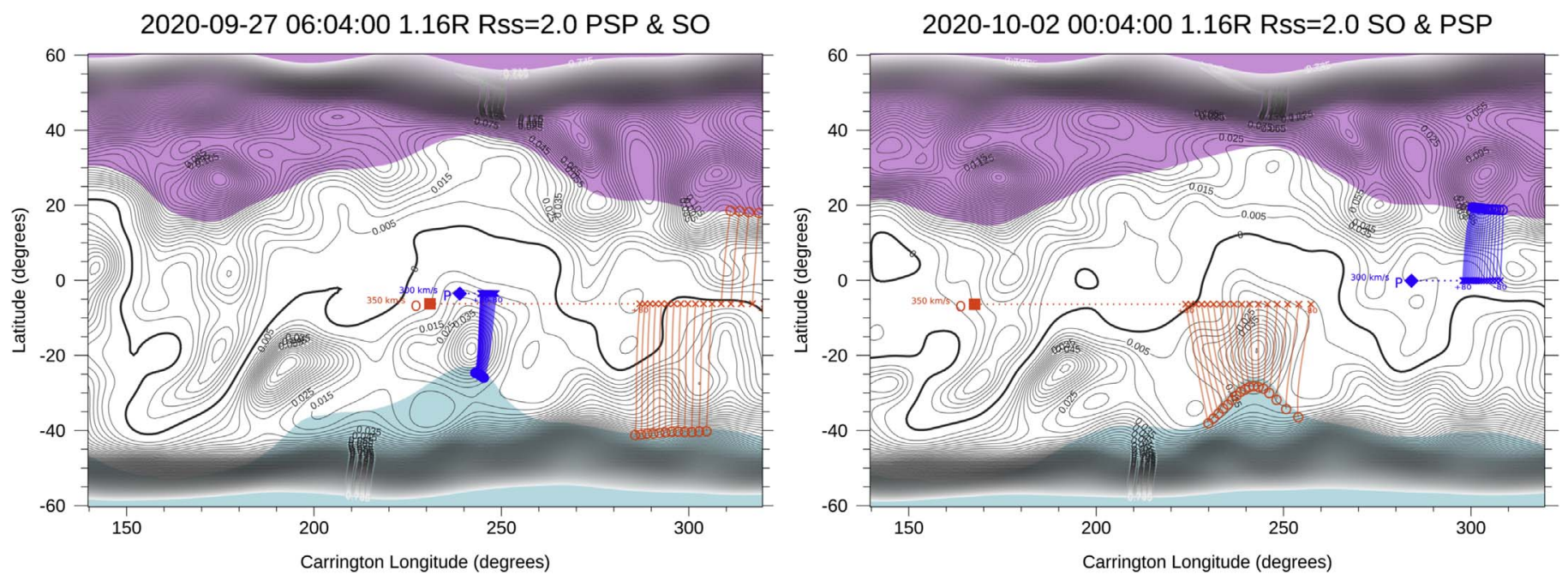

Figure 2. Potential Field Source Surface (PFSS) $|\boldsymbol{B}|^{2}$ contour maps and solar wind magnetic foot-points along the PSP and SolO trajectories for the selected time intervals. The projections of PSP (blue diamonds) and SolO (orange squares) locations on the source surface (blue and orange crosses) and down to the solar wind source region (blue and orange circles), calculated for the height of $1.16 R_{\odot}$ and based on in situ solar wind speed measurements $\pm 80 \mathrm{~km} \mathrm{~s}^{-1}$, are displayed. Open magnetic field regions are shown in blue (negative magnetic polarity) and violet (positive magnetic polarity), and the neutral line is in thick black.

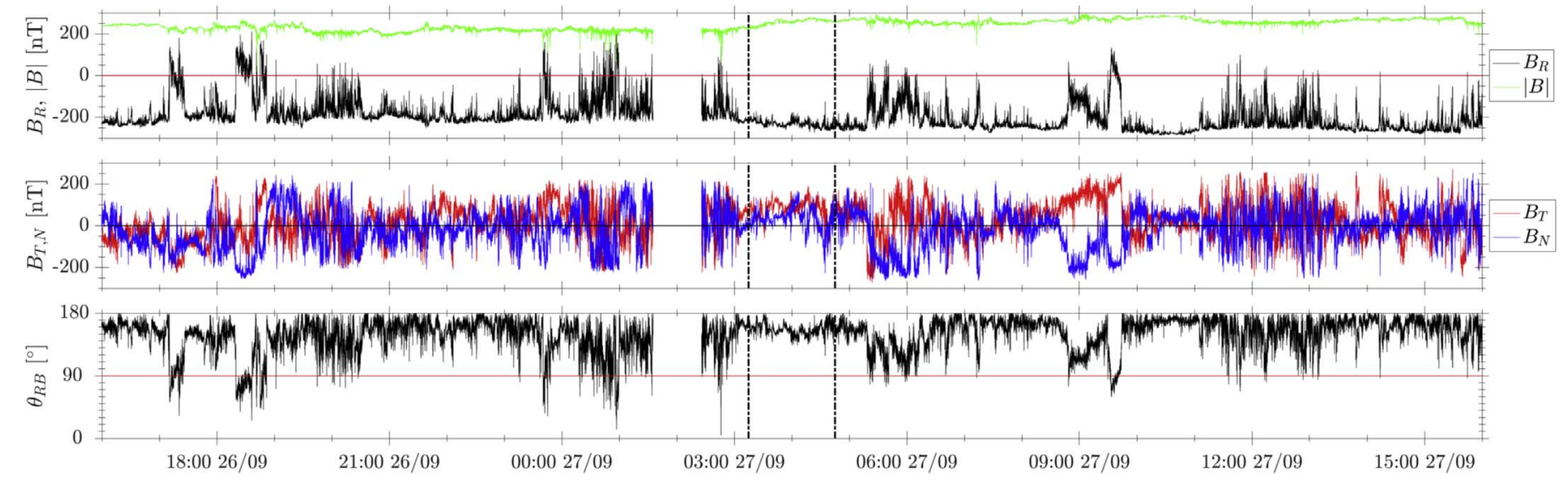

Figure 3. Magnetic field data at PSP in the 1 day interval centered on 2020 September 27 03:15-04:45 (delimited by vertical dashed lines). From top to bottom: $B_{R}$ and $|\boldsymbol{B}|, B_{T}$ and $B_{N}$, and angle $\theta_{R B}$ between the radial direction and $\boldsymbol{B}$.

perihelion, was fast sampling this area, while SolO took a little longer to pass through it due to the natural expansion of the wind and its slower motion. The fact that SolO is magnetically connected to the same region at the Sun as PSP confirms that they sample the same plasma parcel, embedded in an Alfvénic lowspeed stream coming from the same south coronal hole extension.

\section{Evolution of Turbulence from PSP to SolO}

The magnetic field magnitude and components in the heliographic RTN coordinate system, along with the angle between the magnetic field vector and the radial direction $\theta_{R B}=\arccos \left(B_{R} /|\boldsymbol{B}|\right)$, relative to the time periods selected in Section 2 at PSP and SolO, are displayed in the three top panels of Figures 3 and 4, respectively, which overall span a time interval of 1 day. Specifically, PSP/FIELDS $\sim 292 \mathrm{~Hz}$ and SolO/MAG $64 \mathrm{~Hz}$ burst-mode magnetic field data have been used. As expected, because they measure the same plasma parcel, the PSP and SolO periods share common characteristics. Specifically, during both intervals, $|\boldsymbol{B}|$ is quite smooth (revealing that the fluctuations in the wind are largely incompressible) and slightly increasing, the magnetic polarity is negative $\left(B_{R}<0\right)$, and the magnetic field is fairly radial $\left(\theta_{R B} \sim 180^{\circ}\right)$. The observed solar wind plasma hence belongs to a quiescent region, namely a region with exceptionally smooth magnetic field (free of switchbacks) and Parker-spiral-like direction of the magnetic field (Dudok de Wit et al. 2020; Malaspina et al. 2020).

In order to assess the characteristics of turbulence in the plasma parcel observed at two different evolution stages, standard diagnostics for spectral power, compressibility, and intermittency of the magnetic field fluctuations have been used.

The top left panel of Figure 5 shows the spectrum of the magnetic field vector fluctuations $\delta \boldsymbol{B}^{2}$. Power-law fits have been performed at typical fluid scales, between $3 \times 10^{-3}$ and $10^{-1} \mathrm{~Hz}$ in the spacecraft frame, which can be identified as the inertial range of turbulence. The fitted spectral exponents are indicated in the legend. As expected, PSP data shows much larger power, due to the larger amplitude of fluctuations at $0.1 \mathrm{au}$. SolO interval displays a power-law spectrum with scaling exponent $\alpha$ compatible with the standard Kolmogorov fully developed turbulence, $\alpha \sim 5 / 3$ (Kolmogorov 1941). On the contrary, PSP shows a shallower spectrum ( $\alpha \sim 3 / 2$; Chen et al. 2020; Duan et al. 2020), suggesting a less-developed, more Alfvénic turbulence (Bruno \& Carbone 2013), as observed during quiescent intervals (Dudok de Wit et al. 2020; Malaspina et al. 2020). Some caveats are necessary in 


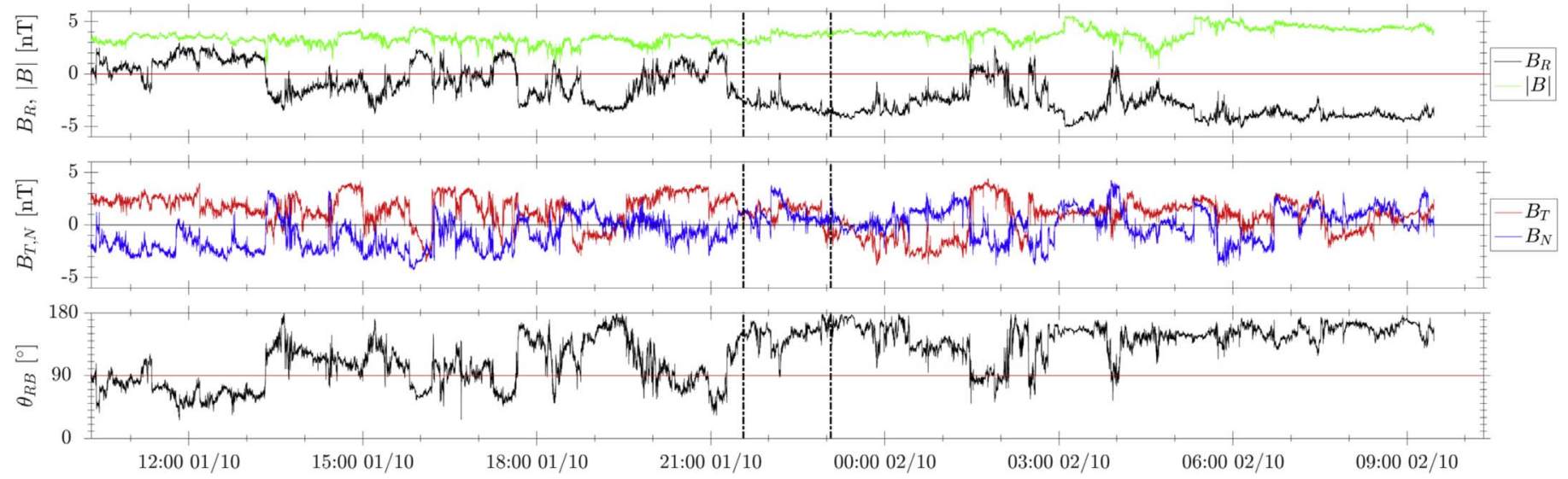

Figure 4. Same as Figure 3, but for 1 day SolO observations centered on the time periods 2020 October 1 21:34-23:04.

interpreting the PSP results since the flow and magnetic fields in the interval of interest are highly aligned (bottom panel of Figure 3), the implication being that advected quasi-2D structures are essentially invisible (Zank et al. 2020) and hence their contribution is difficult to assess. It is worth noting that the power spectrum at $\mathrm{SolO}$ exhibits a high-frequency break around $0.2 \mathrm{~Hz}$, which marks the transition from fluid to kinetic scales. According to previous findings (e.g., Bruno \& Trenchi 2014; Telloni et al. 2019a), this break is close to the proton gyrofrequency, thus suggesting the likely role played by the ion cyclotron resonance mechanism in the spectral cascade.

The highly Alfvénic nature of PSP data was confirmed by the smaller compressibility factor $C=\delta|\boldsymbol{B}|^{2} / \delta \boldsymbol{B}^{2}$ (i.e., the ratio between the spectra of the magnetic field magnitude and vector fluctuations; Bavassano et al. 1982a), shown in the top right panel of Figure 5 and used here as a proxy of Alfvénicity. ${ }^{28}$ Indeed, bearing in mind that the solar wind consists mainly of a mixture of propagating noncompressive Alfvénic fluctuations and compressive magnetic structures advected by the wind, a low magnetic compressibility clearly indicates a dominance of Alfvénic over compressive fluctuations and, in turn, a high Alfvénic content of the solar wind. The turbulent fluctuations observed by SolO are less Alfvénic, in agreement with the known general behavior of the Alfvénicity of tending to decay with increasing distance from the Sun. Such decay could result from nonlinear interactions (Bruno \& Carbone 2013; Zank et al. 2020), transversal velocity and magnetic field shears generated by the solar wind expansion (Shi et al. 2021), or other mechanisms such as parametric decay (e.g., Malara \& Velli 1996; Bruno et al. 2014a). On the other hand, the higher Alfvénicity at PSP may result in stronger decorrelation between the interacting magnetic turbulent structures, reducing the efficiency of the nonlinear cascade (Dobrowolny et al. 1980; Smith et al. 2009; Marino et al. 2011) and leading to a shallower power spectrum.

The presence of intermittency (a characteristic feature of nonlinear turbulence, Anselmet et al. 1984; Frisch 1995; Bruno \& Carbone 2013) was evaluated through the ratio between the fourth and the squared second-order structure functions, i.e., the flatness $\mathcal{F}(1 / f)=\left\langle\Delta \boldsymbol{B}^{4}\right\rangle /\left\langle\Delta \boldsymbol{B}^{2}\right\rangle^{2}$, where $\Delta \boldsymbol{B}(t, 1 / f)$ are increments of

\footnotetext{
28 The Alfvénic character of the fluctuations at PSP is also clearly revealed by the high degree of correlation between magnetic and velocity field fluctuations, i.e., high values of cross-helicity, and by the rather good equipartition between magnetic and kinetic energies, i.e., low values of residual energy (not shown).
}

the magnetic field vector across a timescale $1 / f$ ( $f$ is the frequency). The flatness is typically used to describe deviation from Gaussian-distributed fluctuations (e.g., Frisch 1995; Dudok de Wit et al. 2013; Zhao et al. 2020), for which $\mathcal{F}=3$. This quantity is shown in the bottom left panel of Figure 5. Both intervals show a power-law scaling $\mathcal{F} \sim f^{\beta}$ in the inertial range (approximately from $\sim 3 \times 10^{-3}$ to $\sim 5 \times 10^{-2} \mathrm{~Hz}$ ), as indicated by the thick solid lines in the figure. As a reference, in fluid turbulence $\beta \sim 0.1$ (Frisch 1995), while in space plasmas values between 0.2 and 0.5 have been observed (Sorriso-Valvo et al. 2019; Quijia et al. 2021). The flatness increases slowly at PSP ( $\beta=0.24 \pm 0.03$ ), indicating poorly developed intermittency, and more rapidly at SolO, where the large exponent $(\beta=0.67 \pm 0.06)$ suggests an extremely efficient energy cascade (Carbone \& Sorriso-Valvo 2014). This could be also related to the decrease of the Alfvénic content with distance, allowing the coherent structures advected by the wind to emerge more clearly and control the high-order statistics (Zank et al. 2020).

To ensure statistical significance and ergodicity, the same quantities were also estimated in extended intervals of $2.17 \mathrm{hr}$ for PSP (2020 September 27 03:05-05:15) and $4 \mathrm{hr}$ for SolO (2020 October $121: 20-2020$ October 2 01:20). It is worth noting that the extended intervals also originated at the same solar source (though not strictly corresponding to the same plasma parcel), and the in situ magnetic field characteristics remained approximately stationary. Such a choice also accounts for $\mathrm{a} \pm 5 \mathrm{~km} \mathrm{~s}^{-1}$ uncertainty in the wind bulk speed used for the identification of the plasma parcel (see Section 2). The results obtained in the extended intervals fully confirmed those obtained using the shorter intervals.

Finally, in order to mitigate possible effects of nonstationarity and large-scale structures, intermittency was also assessed using the Hilbert-Huang technique (see, e.g., Huang et al. 1998, 2008; Carbone et al. 2018; Telloni et al. 2019b) to obtain the scaling exponents $\xi_{q}$ of the $q$ th-order structure functions. These were estimated for the magnetic field components and magnitude by resampling and bootstrapping the least-squares fit in the inertial range of the generalized Hilbert spectra, up to the fifth order (Carbone et al. 2020). As shown in the bottom right panel of Figure 5, the deviation from linear order dependence typical of intermittent turbulent flows is observed at SolO for all the magnetic field components and magnitude. Values from a standard experiment of fluid turbulence (Benzi et al. 1993) are shown for comparison. On the other hand, at PSP the scaling exponents are too small, in some cases decreasing with order, and in general do 

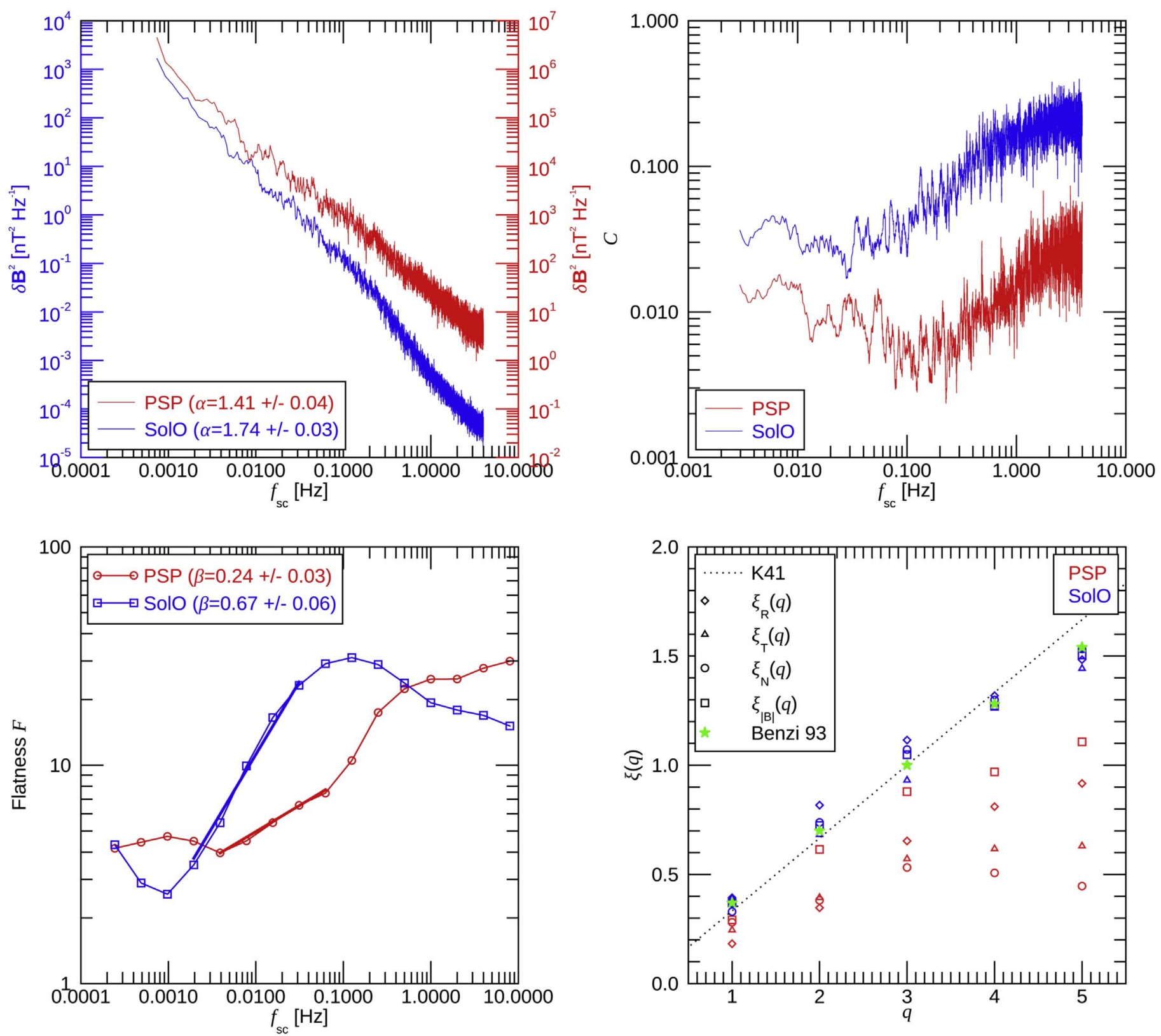

Figure 5. Trace of the magnetic spectral matrix $\delta \boldsymbol{B}^{2}$ (top left), magnetic compressibility spectrum $C$ (top right), and flatness $\mathcal{F}$ as a function of the spacecraft frequency (bottom left) for PSP (red) and SolO (blue) radially aligned intervals. Power-law fits are displayed as thick lines, while relative scaling exponents are reported in the legends. Bottom right: comparison of the scaling exponents $\xi_{q}$ of the $q$ th-order structure functions for PSP (red) and SolO (blue) magnetic field RTN components and magnitude (represented by different symbols as reported in the legend). As a comparison, exponents for velocity fluctuations in the inertial range of hydrodynamic turbulence (green stars; Benzi et al. 1993) and the classical K41 (q/3) Kolmogorov law (dotted line; Kolmogorov 1941) are also displayed.

not compare with standard turbulence (an identified property of the quiescent regions; Dudok de Wit et al. 2020), suggesting once more the poorly developed nature of the turbulent cascade or the presence of strong superposed Alfvénic fluctuations. The secondorder scaling exponent $\xi(2) \equiv \alpha-1$ confirms the spectral observation that the magnetic power has a Kolmogorov scaling for SolO, but shallower scaling for PSP. Similarly, the Hurst number $\mathcal{H}=\xi(1)$ (Flandrin et al. 2004; Carbone et al. 2018), a measure of the memory in the time series (Wang et al. 2000), is much smaller in the PSP interval (mean and standard deviation for the three components being $\mathcal{H} \sim 0.23 \pm 0.05$ ) than for SolO $(\mathcal{H}=0.37 \pm 0.04)$, indicating that the magnetic field at 0.1 au does not show strong memory, which would be expected of intermittent turbulent fluctuations.
The statistical analysis of the intervals at PSP and SolO suggests that the turbulence is evolving during the expansion. Power spectra, intermittency, and compressibility indicate that the nonlinear interactions between Alfvén waves may not yet have had time to fully develop at $0.1 \mathrm{au}$, where the solar wind sampled by PSP is still pristine and unaffected by streamstream interactions. On the contrary, the same plasma has considerably evolved at $1 \mathrm{au}$, where the Alfvénic fluctuations have become smaller. At SolO, the nonlinear interactions might have efficiently formed a fully developed spectrum, additionally building up small-scale intermittent structures and longterm memory. Alternatively, the decrease of the Alfvénic fluctuations could have exposed the intermittent nature of the advected coherent structures. 
Solar Orbiter is a mission of international cooperation between ESA and NASA, operated by ESA. D.T. and R.D.M. were partially supported by the Italian Space Agency (ASI) under contract 2018-30-HH.0. L.S.V. was funded by the Swedish Contingency Agency, grant 2016-2102, and by SNSA, grant86/20. L.D.W. and T.S.H. are supported by the STFC consolidated grant ST/S000364/1. O.P. was supported by the NASA grant 80 NSSC 20 K1829. G.P.Z., M.N., L.A., L. L.Z., and H.L. acknowledge the partial support of a NASA Parker Solar Probe contract SV4-84017, an NSF EPSCoR RIITrack-1 Cooperative Agreement OIA-1655280, and a NASA IMAP grant through SUB000313/80GSFC19C0027. R.L. is supported by an Imperial College President's Scholarship. The French part of this work is supported by CNES, including the support to preparation of PAS data (CDPP/AMDA). The FIELDS and SWEAP teams acknowledge support from NASA contract NNN06AA01C. The Solar Orbiter magnetometer was funded by the UK Space Agency (grant ST/T001062/1). Solar Orbiter Solar Wind Analyser (SWA) work at UCL/MSSL is currently funded under STFC grants ST/T001356/1 and ST/ S000240/1. Solar Orbiter and Parker Solar Probe data were downloaded from the Solar Orbiter Archive (http://soar.esac. esa.int/soar/) and the NASA's Space Physics Data Facility (https://spdf.gsfc.nasa.gov), respectively.

\section{ORCID iDs}

Daniele Telloni $\odot$ https://orcid.org/0000-0002-6710-8142 Luca Sorriso-Valvo @ https://orcid.org/0000-0002-5981-7758 Lloyd D. Woodham (i) https://orcid.org/0000-00032845-4250

Olga Panasenco (i) https://orcid.org/0000-0002-4440-7166 Marco Velli (1) https://orcid.org/0000-0002-2381-3106 Francesco Carbone (1) https://orcid.org/0000-0002-3559-5273 Gary P. Zank (1) https://orcid.org/0000-0002-4642-6192 Roberto Bruno (1) https://orcid.org/0000-0002-2152-0115 Denise Perrone (ㄷ) https://orcid.org/0000-0003-1059-4853 Masaru Nakanotani (i) https://orcid.org/0000-0002-7203-0730 Chen Shi iㅏ https://orcid.org/0000-0002-2582-7085 Raffaella D'Amicis (ํ) https://orcid.org/0000-00032647-117X

Rossana De Marco (1) https://orcid.org/0000-0002-7426-7379 Vamsee K. Jagarlamudi (10) https://orcid.org/0000-00016287-6479

Konrad Steinvall (1) https://orcid.org/0000-0002-5861-1643 Raffaele Marino (i) https://orcid.org/0000-0002-6433-7767

Laxman Adhikari (i) https://orcid.org/0000-0003-1549-5256 Lingling Zhao (1) https://orcid.org/0000-0002-4299-0490 Haoming Liang (ㄷ) https://orcid.org/0000-0001-9581-4821 Anna Tenerani $\odot$ i https://orcid.org/0000-0003-2880-6084 Ronan Laker (i) https://orcid.org/0000-0002-6577-5515 Timothy S. Horbury (1) https://orcid.org/0000-0002$7572-4690$

Stuart D. Bale 주 https://orcid.org/0000-0002-1989-3596

Marc Pulupa (i) https://orcid.org/0000-0002-1573-7457

David M. Malaspina (i) https://orcid.org/0000-00031191-1558

Robert J. MacDowall (1) https://orcid.org/0000-00033112-4201

Keith Goetz iㅏ https://orcid.org/0000-0003-0420-3633 Thierry Dudok de Wit (i) https://orcid.org/0000-00024401-0943

Peter R. Harvey (1) https://orcid.org/0000-0002-6938-0166
Justin C. Kasper (다 https://orcid.org/0000-0002-7077-930X

Kelly E. Korreck (1) https://orcid.org/0000-0001-6095-2490

Davin Larson (1) https://orcid.org/0000-0001-5030-6030

Anthony W. Case (1) https://orcid.org/0000-0002-3520-4041

Michael L. Stevens (다 https://orcid.org/0000-0002-7728-0085

Phyllis Whittlesey (i) https://orcid.org/0000-0002-7287-5098

Roberto Livi (1) https://orcid.org/0000-0002-0396-0547

Christopher J. Owen (i) https://orcid.org/0000-0002-

5982-4667

Stefano Livi (1) https://orcid.org/0000-0002-4149-7311

Philippe Louarn (1) https://orcid.org/0000-0003-2783-0808

Ester Antonucci $\odot$ https://orcid.org/0000-0003-4155-6542

Marco Romoli (16 https://orcid.org/0000-0001-9921-1198

Helen O'Brien (1) https://orcid.org/0000-0002-9833-4097

Vincent Evans (1) https://orcid.org/0000-0003-3732-137X

Virginia Angelini (i) https://orcid.org/0000-0001-6862-2605

\section{References}

Anselmet, F., Gagne, Y., Hopfinger, E. J., et al. 1984, JFM, 140, 63

Bale, S. D., Goetz, K., Harvey, P. R., et al. 2016, SSRv, 204, 49

Bavassano, B., Dobrowolny, M., Fanfoni, G., Mariani, F., \& Ness, N. F. 1982a, SoPh, 78, 373

Bavassano, B., Dobrowolny, M., Mariani, F., \& Ness, N. F. 1981, JGR, 86,1271

Bavassano, B., Dobrowolny, M., Mariani, F., \& Ness, N. F. 1982b, JGR, 87,3617

Benzi, R., Ciliberto, S., Tripiccione, R., et al. 1993, PhRvE, 48, R29

Bruno, R., \& Carbone, V. 2013, LRSP, 10, 2

Bruno, R., Carbone, V., Sorrivo-Valvo, L., \& Bavassano, B. 2003, JGRA, 108,1130

Bruno, R., Telloni, D., Primavera, L., et al. 2014a, ApJ, 786, 53

Bruno, R., \& Trenchi, L. 2014, ApJL, 787, L24

Bruno, R., Trenchi, L., \& Telloni, D. 2014b, ApJL, 793, L15

Carbone, F., \& Sorriso-Valvo, L. 2014, EPJE, 37, 61

Carbone, F., Sorriso-Valvo, L., Alberti, T., et al. 2018, ApJ, 859, 27

Carbone, F., Telloni, D., Sorriso-Valvo, L., et al. 2020, Univ, 6, 116

Chen, C. H. K., Bale, S. D., Bonnell, J. W., et al. 2020, ApJS, 246, 53

Coleman, P. J., Jr. 1968, ApJ, 153, 371

D’Amicis, R., Bruno, R., Pallocchia, G., et al. 2010, ApJ, 717, 474

Dobrowolny, M., Mangeney, A., \& Veltri, P. 1980, PhRvL, 45, 144

Duan, D., Bowen, T. A., Chen, C. H. K., et al. 2020, ApJS, 246, 55

Dudok de Wit, T., Alexandrova, O., Furno, I., Sorriso-Valvo, L., \& Zimbardo, G. 2013, SSRv, 178, 665

Dudok de Wit, T., Krasnoselskikh, V. V., Bale, S. D., et al. 2020, ApJS, 246, 39

Flandrin, P., Rilling, G., \& Goncalves, P. 2004, ISPL, 11, 112

Fox, N. J., Velli, M., Bale, S. D., et al. 2016, SSRv, 204, 7

Frisch, U. 1995, Turbulence (Cambridge: Cambridge Univ. Press)

Goelzer, M. L., Schwadron, N. A., \& Smith, C. W. 2014, JGRA, 119, 115

He, J., Tu, C., Marsch, E., Bourouaine, S., \& Pei, Z. 2013, ApJ, 773, 72

Horbury, T. S., O’Brien, H., Carrasco Blazquez, I., et al. 2020, A\&A, 642, A9

Huang, N. E., Shen, Z., Long, S. R., et al. 1998, RSPSA, 454, 903

Huang, Y. X., Schmitt, F. G., Lu, Z. M., \& Liu, Y. L. 2008, EL, 84, 40010

Kasper, J. C., Abiad, R., Austin, G., et al. 2016, SSRv, 204, 131

Kolmogorov, A. 1941, DoSSR, 30, 301

Malara, F., \& Velli, M. 1996, PhPl, 3, 4427

Malaspina, D. M., Halekas, J., Berčič, L., et al. 2020, ApJS, 246, 21

Marino, R., Sorriso-Valvo, L., Carbone, V., et al. 2011, P\&SS, 59, 592

Müller, D., Cyr, O. C., St., Zouganelis, I., et al. 2020, A\&A, 642, A1

Panasenco, O., Velli, M., D’Amicis, R., et al. 2020, ApJS, 246, 54

Perrone, D., Stansby, D., Horbury, T. S., \& Matteini, L. 2019a, MNRAS, 483, 3730

Perrone, D., Stansby, D., Horbury, T. S., \& Matteini, L. 2019b, MNRAS, 488,2380

Quijia, P., Fraternale, F., Stawarz, J. E., et al. 2021, MNRAS, 503, 4815

Schrijver, C. J., \& De Rosa, M. L. 2003, SoPh, 212, 165

Schwartz, S. J., \& Marsch, E. 1983, JGR, 88, 9919

Shi, C., Velli, M., Panasenco, O., et al. 2021, A\&A, in press (doi:10.1051/ 0004-6361/202039818)

Smith, C. W., Stawarz, J. E., Vasquez, B. J., Forman, M. A., \& MacBride, B. T. 2009, PhRvL, 103, 201101 
Sorriso-Valvo, L., De Vita, G., Fraternale, F., et al. 2019, FrP, 7, 108 Telloni, D., Bruno, R., \& Trenchi, L. 2015, ApJ, 805, 46

Telloni, D., Carbone, F., Bruno, R., et al. 2019a, ApJL, 885, L5

Telloni, D., Carbone, F., Bruno, R., et al. 2019b, ApJ, 887, 160

Tu, C.-Y., \& Marsch, E. 1995, SSRv, 73, 1

Velli, M., Harra, L. K., Vourlidas, A., et al. 2020, A\&A, 642, A4
Wang, G., Antar, G., \& Devynck, P. 2000, PhPl, 7, 1181

Zank, G. P., Adhikari, L., Hunana, P., et al. 2017, ApJ, 835, 147

Zank, G. P., Nakanotani, M., Zhao, L.-L., Adhikari, L., \& Telloni, D. 2020, ApJ, 900, 115

Zhao, L.-L., Zank, G. P., Adhikari, L., et al. 2020, ApJ, 898, 113

Zouganelis, I., De Groof, A., Walsh, A. P., et al. 2020, A\&A, 642, A3 